Bull. Austral. Math. Soc.

VOL. 67 (2003) [407-411]

\title{
ON INCIDENCE RINGS OF GROUP AUTOMATA
}

\author{
A.V. KeLAREV
}

For all finite $p$-groups, we describe the Jacobson radicals of incidence rings of group automata.

We use standard concepts of automata and languages theory following [2] and [7]. The reader is referred to $[3,4]$ for preliminaries on ring constructions and their applications. Incidence rings of graphs have been actively investigated by many authors and it turns out that this concept provides valuable information on the related classes of graphs $($ see $[3,8])$. It is natural to expect that an analogue of this construction may turn out to be useful in automata and languages theory. We consider a generalisation of incidence rings of graphs defined for all finite group automata. A finite group automaton is an algebraic system $\mathcal{A}=(Q, G, \delta)$, where

1. $Q$ is a finite set of states;

2. $G$ is a finite group of input symbols;

3. $\delta: Q \times G \rightarrow Q$ is a transition function satisfying the equality

$$
\delta(q, g h)=\delta(\delta(q, g), h)
$$

for all $q \in Q, g, h \in G$ (see [6]).

Notation $q g=q \cdot g$ is also used for $\delta(q, g)$, where $q \in Q, g \in G$. Group automata are worth considering since they occur in the Krohn-Rhodes Decomposition Theorem if we identify words of the free monoid on the input alphabet with their images in the transition group (see [2], [4, Section 29], and [6]). It is possible to record this definition for any semigroup automaton and allow not only functions but also relations to be involved as $\delta$ in order to embrace nondeterministic automata. Our definition has been stated in the more special case, because in the present paper we concentrate on group automata only.

By analogy with incidence rings of graphs we introduce the concept of an incidence ring of an automaton. Let $R$ be a ring. The incidence ring of the automaton $\mathcal{A}$ over $R$ is the ring $I_{\mathcal{A}}(R)$ which is spanned as a left $R$-module by the set $T_{\mathcal{A}}$ of all triples $(g ; q, q g)$,

Received 9th September, 2002

Copyright Clearance Centre, Inc. Serial-fee code: 0004-9727/03 \$A2.00+0.00. 
where $g \in G, q \in Q$, and is equipped with multiplication defined by the distributive law and the rules

$$
\begin{aligned}
\left(g_{1} ; q_{1}, q_{1} g_{1}\right) \cdot\left(g_{2} ; q_{2}, q_{2} g_{2}\right) & = \begin{cases}\left(g_{1} g_{2} ; q_{1}, q_{2} g_{2}\right) & \text { if } q_{2}=q_{1} g_{1} \\
0 & \text { otherwise }\end{cases} \\
r \cdot\left(g_{1}, q_{1}, q_{1} g_{1}\right) & =\left(g_{1}, q_{1}, q_{1} g_{1}\right) \cdot r,
\end{aligned}
$$

for all $g_{1}, g_{2} \in G, q_{1}, q_{2}, \in Q, r \in R$.

The Jacobson radical is one of the major tools used in the investigation of the structure of ring constructions (see $[1,3,5]$ for references). Our main theorem gives a complete description of the Jacobson radicals of incidence rings of group automata for all finite $p$-groups.

The Jacobson radical of a ring $R$ is denoted by $J(R)$. Let $N_{\mathcal{A}}$ be the set of all triples $(g ; q, q g) \in T_{\mathcal{A}}$ such that $q \notin Q e$, where $e$ is the identity of the group. As usual, denote by $F\left[N_{\mathcal{A}}\right]$ the set of all finite sums $\sum_{i=1}^{m} f_{i} t_{i}$, where $f_{i} \in F, t_{i} \in N_{\mathcal{A}}$. For any triple $u=(g ; q, q g) \in T_{\mathcal{A}}$, denote by $\widehat{u}$ the pair $(q, q g)$. If $R$ is a ring, then we put

$$
\omega\left(I_{\mathcal{A}}(R)\right)=\sum_{u, v \in T_{\mathcal{A}}, \widehat{u}=\widehat{v}} R(u-v) .
$$

TheOREM 1. Let $F$ be a field of characteristic $p$, and let $\mathcal{A}=(Q, G, \delta)$ be a finite group automaton, where $G$ is a p-group for a prime $p$. Then the Jacobson radical of the incidence ring $I_{\mathcal{A}}(F)$ is equal to

$$
J\left(I_{\mathcal{A}}(F)\right)=F\left[N_{\mathcal{A}}\right]+\omega\left(I_{\mathcal{A}}(F)\right) .
$$

The following useful lemma is well known; in particular, it follows from the modular law that holds in the lattice of ideals in every ring.

Lemma 2. Let $R$ be a ring with ideals $A, B, I$ such that $A \subseteq B$. If $A+I=B+I$ and $A \cap I=B \cap I$, then $A=B$.

If $R$ is a ring and $G$ is a semigroup, then the semigroup ring $R[G]$ consists of all sums of the form $\sum_{g \in G} r_{g} g$, where $r_{g} \in R$ for all $g \in G$, and only a finite number of the coefficients $r_{g}$ are nonzero, with addition and multiplication defined by the rules

$$
\begin{aligned}
\sum_{g \in G} r_{g} g+\sum_{g \in G} r_{g}^{\prime} g & =\sum_{g \in G}\left(r_{g}+r_{g}^{\prime}\right) g, \\
\left(\sum_{g \in G} r_{g} g\right)\left(\sum_{h \in G} r_{h}^{\prime} h\right) & =\sum_{g, h \in G}\left(r_{g} r_{h}^{\prime}\right) g h .
\end{aligned}
$$

If $G$ is a group, then $F[G]$ is called a group ring. If $S$ is a semigroup with zero $\theta$, then the quotient ring $F[S] / F \theta$ is called a contracted semigroup ring, and is denoted by $F_{0}[S]$. 
Straightforward verification shows that $T_{\mathcal{A}}^{0}=T_{\mathcal{A}} \cup\{0\}$ is a semigroup and $I_{\mathcal{A}}(F)$ is an associative ring isomorphic to the contracted semigroup ring $F_{0}\left[T_{A}^{0}\right]$.

We also need the following two known lemmas (see [3]).

Lemma 3. Let $F$ be a field of characteristic $p$, and let $G$ be a finite $p$-group. Then the Jacobson radical $J(F[G])$ is equal to the augmentation ideal

$$
\omega(F[G])=\left\{\sum_{i=1}^{n} f_{i} g_{i} \mid f_{i} \in F, g_{i} \in G, \sum_{i=1}^{n} f_{i}=0\right\} .
$$

LEMmA 4. If $R$ is a ring and $M_{n}(R)$ is the ring of $n \times n$ matrices over $R$, then

$$
J\left(M_{n}(R)\right)=M_{n}(J(R)) .
$$

Proof of Theorem 1. First, we claim that $N_{\mathcal{A}}^{0}=N_{\mathcal{A}} \cup\{0\}$ is a nilpotent ideal of $T_{\mathcal{A}}^{0}$. Indeed, choose any $\left(g_{1} ; q_{1}, q_{1} g_{1}\right) \in T_{\mathcal{A}}$ and $\left(g_{2} ; q_{2}, q_{2} g_{2}\right) \in N_{\mathcal{A}}$. By the definition of $N_{\mathcal{A}}$, we get $q_{2} \notin Q e$. Since $q_{1} g_{1}=\left(q_{1} g_{1}\right) e \in Q e$, it follows that $q_{1} g_{1} \neq q_{2}$, and therefore $\left(g_{1} ; q_{1}, q_{1} g_{1}\right)\left(g_{2} ; q_{2}, q_{2} g_{2}\right)=0$ by (2). Hence $T_{\mathcal{A}} N_{\mathcal{A}}=0$. Therefore $\left(F\left[N_{\mathcal{A}}\right]\right)^{2}=0$. Since $F\left[N_{\mathcal{A}}\right]$ is an ideal of $F_{0}\left[T_{\mathcal{A}}^{0}\right]$, it follows that $F_{0}\left[N_{\mathcal{A}}^{0}\right] \subseteq J\left(F_{0}\left[T_{\mathcal{A}}^{0}\right]\right)=J\left(I_{\mathcal{A}}(F)\right)$.

Next, we define a mapping $f$ from $I_{\mathcal{A}}(F)$ into the matrix ring $M_{n}(F[G])$ over the group ring $F[G]$, where $n=|Q|$ is the number of states of the automaton $\mathcal{A}$. To this end let us identify the states of $\mathcal{A}$ with integers $1,2, \ldots, n$ so that all columns and all rows of every $n \times n$ matrix ring become labelled by the elements of $Q$. Denote by $e_{r, c}$ the standard elementary matrix with 1 in the intersection of row $r$ and column $c$, where $r, c \in Q$, and with zeros in all other entries. Then $M_{n}(F)=\bigoplus_{r, c \in Q} F e_{r, c}$. For each $x=\sum_{i=1}^{m} f_{i}\left(g_{i} ; q_{i}, q_{i} g_{i}\right) \in I_{\mathcal{A}}(F)$, where $f_{i} \in F,\left(g_{i} ; q_{i}, q_{i} g_{i}\right) \in T_{\mathcal{A}}$, we set ${ }^{r}$

$$
f\left(\sum_{i=1}^{m} f_{i}\left(g_{i} ; q_{i}, q_{i} g_{i}\right)\right)=\sum_{i=1}^{m}\left(f_{i} g_{i}\right) e_{q_{i}, q_{i} g_{i}} \in M_{n}(F[G]) .
$$

A tedious but routine verification shows that this mapping is a monomorphism,

$$
f\left(\omega\left(I_{\mathcal{A}}(F)\right)\right) \subseteq M_{n}(\omega(F[G])),
$$

and besides

$$
f^{-1}\left(M_{n}(\omega(F[G]))\right)=\omega\left(I_{\mathcal{A}}(F)\right) .
$$

Since $F[G] / \omega(F[G]) \cong F$, it follows from (7) that

$$
M_{n}(F[G]) / M_{n}(\omega(F[G])) \cong M_{n}(F)
$$

and so we may identify $M_{n}(F)$ with $M_{n}(F[G]) / M_{n}(\omega(F[G]))$. 
Lemma 3 tells us that $J(F[G])=\omega(F[G])$. The Jacobson radical of every finite dimensional algebra is nilpotent, and hence it follows that $\omega(F[G])$ is nilpotent, and so the same can be said of $M_{n}(\omega(F[G]))$. Therefore the subring $f\left(\omega\left(I_{\mathcal{A}}(F)\right)\right)$ of $M_{n}(\omega(F[G]))$ is nilpotent too. Since $f$ is injective, we see that $\omega\left(I_{\mathcal{A}}(F)\right)$ is a nilpotent ideal of $I_{\mathcal{A}}(F)$; whence $\omega\left(I_{\mathcal{A}}(F)\right) \subseteq J\left(I_{\mathcal{A}}(F)\right)$. Thus we have proved that

$$
F\left[N_{\mathcal{A}}\right]+\omega\left(I_{\mathcal{A}}(F)\right) \subseteq J\left(I_{\mathcal{A}}(F)\right)
$$

Consider the quotient ring $R=I_{\mathcal{A}}(F) / \omega\left(I_{\mathcal{A}}(F)\right)$. Let $\varphi$ be the natural homomorphism from $I_{\mathcal{A}}(F)$ to $R$ with kernel $\omega\left(I_{\mathcal{A}}(F)\right.$ ). Conditions (6) and (7) show that $f$ induces an isomorphic embedding $\psi$ of $R$ into $M_{n}(F)$. For every $q \in Q$, denote by $D_{q}=\left(V_{q}, E_{q}\right)$ the subgraph induced by the set $q G$ in the graph $D=(Q, E)$, where

$$
E=\{(q, q g) \mid q \in Q, g \in G\}
$$

It is easily seen each $D_{q}$ is a maximal complete subgraph of $D$. Hence it follows that the image $\psi\left(R_{q}\right)$ of the ring

$$
R_{q}=\sum_{g, h \in G} F \varphi(h ; q g, q g h)
$$

contains all standard elementary matrices $e_{r, c}$ with $r, c \in V_{q}$, and so $R_{q}$ is isomorphic to the full matrix ring of $|q G| \times|q G|$ matrices over $F$.

It is straightforward to verify that $D$ is the disjoint union of $N_{\mathcal{A}}$ and the complete subgraphs $D_{q}$, for $q \in Q$. Therefore $R$ is a direct sum of the ideal $F\left[N_{\mathcal{A}}\right]$ and full matrix rings $R_{q}, q \in Q$. It follows that $J(R)=F\left[N_{\mathcal{A}}\right]$, and Lemma 2 implies $J\left(I_{\mathcal{A}}(F)\right)$ $=F\left[N_{\mathcal{A}}\right]+\omega\left(I_{\mathcal{A}}(F)\right)$, as required.

An associative ring $R$ is called a $G$-graded ring if $R=\bigoplus_{g \in G} R_{g}$ is a direct sum of additive groups $R_{g}$, and $R_{g} R_{h} \subseteq R_{g h}$ for all $g, h \in G$. It is easily seen that $I_{\mathcal{A}}(F)$ is a $G$-graded ring:

$$
I_{\mathcal{A}}(R)=\bigoplus_{g \in G} R_{g}, \text { where } R_{g}=\bigoplus_{(g ; q, q g) \in T_{\mathcal{A}}} R(g ; q, q g) \text { for } g \in G .
$$

The following example of a group automaton $\mathcal{A}=\left(\{1,2,3\},\left\{e, g, g^{2}\right\}, \delta\right)$ with transition diagram in Figure 1 illustrates this.

The incidence ring of this automaton is graded as follows:

$$
\begin{aligned}
I_{\mathcal{A}}(F) & =R_{e}+R_{g}+R_{g^{2}} \\
& =\left[\begin{array}{lll}
F & 0 & 0 \\
0 & F & 0 \\
0 & 0 & F
\end{array}\right] e+\left[\begin{array}{lll}
0 & F & 0 \\
0 & 0 & F \\
F & 0 & 0
\end{array}\right] g+\left[\begin{array}{lll}
0 & 0 & F \\
F & 0 & 0 \\
0 & F & 0
\end{array}\right] g^{2}
\end{aligned}
$$




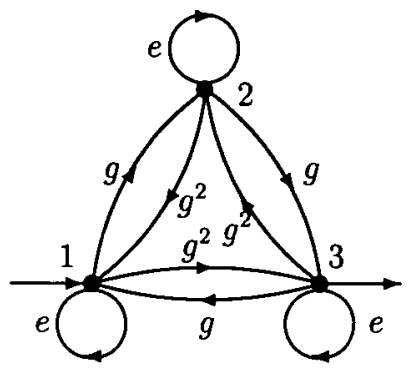

Figure 1: A group automaton $\mathcal{A}=\left(\{1,2,3\},\left\{e, g, g^{2}\right\}, \delta\right)$.

\section{REFERENCES}

[1] B.J. Gardner, Radical theory, Pitman Research Notes in Mathematics 198 (Longman Scientific \& Technical, Harlow, 1989).

[2] M. Ito, Algebraic theory of automata and languages (World Scientific,, 2001).

[3] A.V. Kelarev, Ring constructions and applications, Series in Algebra 9 (World Scientific, River Edge, N.J., 2002).

[4] R. Lidl and G. Pilz, Applied abstract algebra (Springer-Verlag, New York, 1998).

[5] D.S.Passman, The algebraic structure of group rings (J. Wiley \& Sons, London, Sydney, 1977).

[6] B.I. Plotkin, L.Ja. Greenglaz and A.A. Gvaramija, Algebraic structures in automata and databases theory (World Scientific, River Edge, N.J., 1992).

[7] G. Rozenberg and A. Salomaa (Editors), Handbook of formal languages, Vol. 1, 2, 3, (Springer-Verlag, Berlin, 1997).

[8] E. Spiegel and C.J. O'Donnell, Incidence algebras, Monographs and Textbooks in Pure and Applied Mathematics 206 (Marcel Dekker, New York, 1997).

Department of Mathematics

University of Tasmania

G.P.O. Box 252-37

Hobart

Tasmania 7001

Australia

e-mail: Andrei.Kelarev@utas.edu.au 